\author{
Bastiaan H. Wittekamp \\ David S. Y. Ong \\ Olaf L. Cremer \\ Marc J. M. Bonten
}

\section{Nystatin versus amphotericin B to prevent and eradicate Candida colonization during selective digestive tract decontamination in critically ill patients}

Published online: 12 October 2015

(C) Springer-Verlag Berlin Heidelberg and ESICM 2015

B. H. Wittekamp and D. S. Y. Ong contributed equally to this work as co-first authors.

Electronic supplementary material

The online version of this article (doi: 10.1007/s00134-015-4081-x) contains supplementary material, which is available to authorized users.

Dear Editor,

Selective digestive tract decontamination (SDD) and selective oropharyngeal decontamination (SOD) aim to eliminate potential pathogenic microorganisms, such as Gram-negative bacteria and yeasts, from the oropharynx and digestive tract of intensive care unit (ICU) patients [1-3]. Amphotericin B is mostly used as the antifungal component in SOD and SDD, but is increasingly difficult to acquire due to scarcity of raw materials and is also becoming increasingly expensive. Nystatin is a potential substitute as it is also effective against a broad range of fungi and not absorbed from the digestive tract [4]. However, the effects of both agents on Candida colonization and eradication in SDD have never been compared.

We performed a before-after study evaluating two subsequent changes in SDD in a 32-bed medical-surgical ICU in the Netherlands. All patients admitted for $48 \mathrm{~h}$ or longer (i.e., eligible to receive SDD until discharge from the ICU) and who did not receive systemic antifungal therapy were included in the analysis. The local ethics committee waived the need for informed consent.
There were three study periods (Table S1): period 1 (16 months), SDD treatment included (q.d.s.) application of a mouth paste containing $2 \%$ polymyxin E, $2 \%$ tobramycin and $2 \%$ amphotericin $\mathrm{B}$, administration (q.d.s) of a suspension with the same components $(100 \mathrm{mg}$ polymyxin $\mathrm{E}, 80 \mathrm{mg}$ tobramycin and $500 \mathrm{mg}$ amphotericin B) through the nasogastric tube, and the systemic administration (q.d.s.) of cefotaxime during the first 4 days of ICU admission $(\mathrm{Am} / \mathrm{Am})$; period 2 (17 months), nystatin $\left(2 \times 10^{\wedge} 6\right.$ units per dose) replaced amphotericin B in the enteral solution only (Am/Nys); period 3 (10 months), nystatin replaced amphotericin B in both the oropharyngeal paste and enteral solution (Nys/Nys).

From 1468 patients at least two rectum surveillance cultures were available, 1095 (75 \%) were not colonized at the start of ICU admission and Candida acquisition in the rectum was analyzed. Patients' characteristics are on Table S2. Compared to the reference period (i.e., $\mathrm{Am} / \mathrm{Am}$ ) and after adjustment for baseline imbalances in a Cox regression analysis,

Table 1 Effectiveness of nystatin versus amphotericin B in preventing Candida acquisition and achieving decolonization

\begin{tabular}{|c|c|c|c|}
\hline & $\begin{array}{l}\text { Number of events/number } \\
\text { of patients }\end{array}$ & $\begin{array}{l}\text { Crude hazard ratio } \\
(95 \% \mathrm{CI})\end{array}$ & $\begin{array}{l}\text { Adjusted hazard ratio } \\
(95 \% \mathrm{CI})\end{array}$ \\
\hline \multicolumn{4}{|l|}{ Candida acquisition in rectum } \\
\hline $\mathrm{Am} / \mathrm{Am}$ (reference group, period 1) & $80 / 441$ & 1 & 1 \\
\hline Am/Nys (period 2) & $62 / 415$ & $0.75(0.54-1.05)$ & $0.74(0.53-1.03)^{\mathrm{a}}$ \\
\hline Nys/Nys (period 3) & $24 / 239$ & $0.53(0.33-0.83)$ & $0.52(0.33-0.83)^{\mathrm{a}}$ \\
\hline \multicolumn{4}{|l|}{ Candida acquisition in sputum } \\
\hline Am/Am (reference group, period 1) & $61 / 295$ & 1 & 1 \\
\hline Am/Nys (period 2) & $79 / 215$ & $1.23(0.88-1.72)$ & $1.21(0.86-1.70)^{\mathrm{b}}$ \\
\hline Nys/Nys (period 3) & $31 / 156$ & $0.87(0.57-1.33)$ & $0.85(0.55-1.31)^{\mathrm{b}}$ \\
\hline \multicolumn{4}{|l|}{ Candida decolonization in rectum } \\
\hline $\mathrm{Am} / \mathrm{Am}$ (reference group, period 1) & $59 / 127$ & 1 & 1 \\
\hline Am/Nys (period 2) & $84 / 150$ & $1.23(0.88-1.72)$ & $1.22(0.87-1.70)^{\mathrm{c}}$ \\
\hline Nys/Nys (period 3) & $57 / 96$ & $1.71(1.19-2.48)$ & $1.70(1.18-2.45)^{\mathrm{c}}$ \\
\hline
\end{tabular}
equivalent), chemotherapy/radiotherapy in the year preceding intensive care unit admission, and a known humoral or cellular immune deficiency 
the hazard ratios (HR) for acquisition Compliance with ethical standards of Candida colonization in the intestinal tract were $0.74(95 \%$ CI 0.53-1.03) during Am/Nys and 0.52 (95\% CI 0.33-0.83) during Nys/Nys (Table 1). After adjustment for baseline imbalances, the HR for decolonization of Candida in the rectum was 1.70 (95\% CI 1.18-2.45) during Nys/Nys (Table 1). In the 1378 patients with at least two sputum surveillance cultures available, acquisition rates of Candida in the respiratory tract were not significantly different in the three study periods (Table 1).

None of the included patients acquired candidemia, and the numbers of patients with at least one episode of bacteremia were 15.0, 14.2 and 17.7 per 1000 patient days for period 1,2 and 3 , respectively (Table S1).

In SDD, nystatin was more effective than amphotericin B in eradicating Candida from the rectum and preventing rectal Candida colonization, and is not inferior to amphotericin B in preventing Candida respiratory tract colonization. Nystatin use will improve the costeffectiveness of SDD and SOD.
Conflicts of interest BHJW and MJMB are supported by funding from the European Community's Seventh Framework Programme (FP7/2007-2013) under grant agreement 282512. The authors declare that they have no conflict of interest.

\section{References}

1. de Smet AM, Kluytmans JA, Cooper BS, Mascini EM, Benus RF, van der Werf TS et al (2009) Decontamination of the digestive tract and oropharynx in ICU patients. N Engl J Med 360:20-31

2. de Jonge E, Schultz MJ, Spanjaard L, Bossuyt PM, Vroom MB, Dankert J et al (2003) Effects of selective decontamination of digestive tract on mortality and acquisition of resistant bacteria in intensive care: a randomised controlled trial. Lancet 362:1011-1016

3. Ong DS, Klein Klouwenberg PM, Spitoni C, Bonten MJ, Cremer OL (2013) Nebulised amphotericin B to eradicate Candida colonisation from the respiratory tract in critically ill patients receiving selective digestive decontamination: a cohort study. Crit Care 17:R233
4. Eggimann P, Wolff M, Garbino J (2005) Oral nystatin as antifungal prophylaxis in critically ill patients: an old SDD tool to be renewed? Intensive Care Med 31:1466-1468

B. H. Wittekamp · D. S. Y. Ong ·

M. J. M. Bonten

Julius Center for Health Sciences and

Primary Care, University Medical Center

Utrecht, Utrecht, The Netherlands

D. S. Y. Ong · O. L. Cremer

Department of Intensive Care Medicine, University Medical Center Utrecht, Utrecht, The Netherlands

D. S. Y. Ong - M. J. M. Bonten

Department of Medical Microbiology, University Medical Center Utrecht, Utrecht, The Netherlands

D. S. Y. Ong $(\bowtie)$

G04.614 Postbus 85500, 3508 GA Utrecht, The Netherlands

e-mail: davidsyong@gmail.com

Tel.: 0031 (0)88 7558108 